\title{
Preparation, Characterization and Anti- bacterial Activity of Chitosan/Carbon Nanotube Nanocomposites
}

\author{
Abeer Abdulaziz H. Bukhari* \\ Department of Chemistry, Faculty of Science, University of Tabuk, Saudi Arabia; a.bukhari@ut.edu.sa
}

\begin{abstract}
Objective: To prepare Chitosan-Multi Walled Carbon Nanotube (Ch: MWCNT) nanocomposite with different chitosan: MWCNT ratios were successfully prepared by modifying chitosan and functionalized MWCNT. Methods: The prepared Ch-MWCNTs nanocomposite was characterized by TEM analysis confirming the MWCNTs homogenously distributed in chitosan matrix. Furthermore, TEM results indicate the resulting MWCNT tubular length morphology. Based on the FTIR results which confirm the presence of the $\mathrm{C}=\mathrm{C}$. The FTIR indicates that the characteristic functional groups of MWCNTs and chitosan are successfully present in modified nanocomposite. Findings/Application: Nanocomposite Ch/MWCNT showed higher antimicrobial activity against both Gram negative and Gram positive bacteria. The nanocomposites are highly differentiable at the lower conc. as even only $1 \%$ conc. from multifunctional nanocomposite has found to be very effective against the target microorganisms. Minimal Inhibitory Concentrations (MIC) of Ch-MWCNT (100:50 Ch/MWCNT) against Gram negative and Gram positive pathogenic bacteria between 0.5 and $0.0625 \mu \mathrm{l}$, and the growth inhibition effect has been observed in a concentration-dependent species.
\end{abstract}

Keywords: Antimicrobial, Chitosan, CNT, Nanocomposites

\section{Introduction}

Since their discovery in by ${ }^{1}$, the CNTs have received much attention in many scientific fields. The remarkable electronic properties of CNTs, their sensitivity to the external environment and their nanoscale dimension, make them very attractive compounds for biosensors for the detection of biomolecules. Thus, the biosensors based on CNTs developed so far can detect proteins, glucose, DNA and urea ${ }^{2-4}$.

A key point for multi-walled carbon nanotubes to be able to envisage an industrial exploitation is the development of a technique of production which makes it possible to obtain large quantities of material with the controlled morphology and at a competitive price, while maintaining high purity to avoid possible problems of toxicity and ecotoxicity. Research is still underway to solve the problem of CNT dispersion in both organic solvents and polymer matrices. Indeed, their covalent and non-covalent functionalization, using new alternatives, may be the most appropriate solution to broaden their application. The functionalization of the CNTs by modification of their surface renders the biocompatible CNTs to become fluorescent and used in imaging, or to convey an active principle specifically towards biological targets. On the other hand, the use of a natural polymer has been also favored, because of its natural biocompatibility and also for ecological reasons.

Chitosan has received much attention for its interesting properties such as nontoxicity, biocompatibility and biodegradability. It can be degraded into non-toxic compounds and be metabolized by enzymes in human body

*Author for correspondence 
like lysozyme. Thus, chitosan is soluble in weak acids has a high potential because of its poly cationic character that distinguishes it from other polysaccharides and natural polymers that are generally anionic. In addition, chitosan is mucoadhesive and acts as an antibacterial agent, which makes it possible to use in wound healing ${ }^{5,6}$.

In the present paper Chitosan-Multi Walled Carbon Nanotube (Ch:MWCNT) nanocomposite has been prepared, characterized and tested against Gram negative and Gram positive pathogenic bacteria.

\section{Materials and Methods}

Multi Wall Carbon Nanotube (MWCNT, Outer diameter: 7-15 nm, Length: $0.5-10 \mathrm{~mm}$ ) were purchased from Sigma-Aldrich. $\mathrm{HNO}_{3}(67 \%)$ and $\mathrm{H}_{2} \mathrm{SO}_{4}(98 \%)$ were purchased from Nen Tech Ltd. Brixworth Northants. U.K. Chitosan was purchased from Mallinckrodt, USA [M.W $=400,000$, degree of deacetylation 95\%].

The tested organisms included Gram negative bacteria (Escherichia coli O157H7 and Pseudomonas aeruginosa ATCC; 10145) and Gram positive bacteria (Staphylococcus aureus MRSA; 23516 and Methicillinresistant Staphylococcus aureus MRSA; 629012).

\section{Composition of media used:}

- Nutrient agar ${ }^{7}$

\begin{tabular}{|l|l|}
\hline Ingredients & g liter $^{-1}$ \\
\hline Beef extract & $3.0 \mathrm{~g}$ \\
\hline Peptone & $5.0 \mathrm{~g}$ \\
\hline Agar & $15.0 \mathrm{~g}$ \\
\hline Distilled water & $1000 \mathrm{ml}$ \\
\hline $\mathrm{pH}$ & 7.2 \\
\hline
\end{tabular}

- Luria Bertani (LB) broth Medium ${ }^{8}$

\begin{tabular}{|l|l|}
\hline Ingredients & gliter $^{-1}$ \\
\hline $\mathrm{NaCl}$ & 10.0 \\
\hline Tryptone & 10.0 \\
\hline Yeast extract & 5.0 \\
\hline Distilled water & $1000 \mathrm{ml}$ \\
\hline $\mathrm{pH}$ & 7.5 \\
\hline
\end{tabular}

\subsection{Preparation of Bacterial Inoculums}

All test bacteria were grown in Luria-Bertani (LB) medium in presence of $20 \%$ Agar $\stackrel{8}{-}$ and incubated at $37^{\circ} \mathrm{C}$ for $24 \mathrm{hrs}$ and a total of $10 \mathrm{ml}$ of the pure culture was centrifuged to pellet out the cells, washed twice with sterile physiological saline solution and the suspension was adjusted to optical density 0.1 at $600 \mathrm{~nm}$ which is equivalent to a cell population of about $10^{6}$ cells $/ \mathrm{ml}$ on the McFarland standard. Bacteria suspension was stored in test tubes in a refrigerator at $4^{\circ} \mathrm{C}$. These test bacteria were frequently checked for viability and prepared when appropriate.

\subsection{Antibacterial Activity Test}

Antibacterial activity test was performed using disc diffusion method? ${ }^{9}$ LB agar plates were prepared, sterilized and solidified. After solidification pathogenic microorganisms cultures were swabbed on these plates. The sterile discs were dipped in the chitosan compound solutions and placed on the surface of the agar plates and kept for incubation at $37^{\circ} \mathrm{C}$ for $24 \mathrm{hrs}$. The diameter of zone of inhibition was measured. Zone of inhibition was measured and compared with control disc. The negative control treats with solvent (acetic acid) and positive control with Nalidixic acid. The experiments were repeated three times and mean values of zone diameters were recorded. Compound showing promising activities was selected for further studies.

\subsection{Minimal Inhibitory Concentrations (MIC)}

MIC was performed using nutrient Agar via serial dilution method ${ }^{10}$. Approximately $1 \mathrm{mg}$ of the selected promising activity Ch-MWCNT nanocomposite was weighted and dissolved in $100 \mu \mathrm{l}$ of $100 \%$ acetic acid. A stock solution was diluted to concentration of 1:2, 1:4, 1:8 and 1:16, each tested in triplicate, respectively. With a precision delivery system, absorbent paper disks $(6 \mathrm{~mm})$ were individually impregnated with $10 \mu \mathrm{l}$ of each concentration of the selected promising activity Ch-MWCNT agent. The disks were immediately subjected to rapid drying and then placed on the surface of the agar plates inoculated with tested pathogenic organisms and incubated at $37^{\circ} \mathrm{C}$ for 24 hrs. The Minimum Inhibitory Concentration (MIC) was regarded as the lowest concentration of the Ch-MWCNT required to completely inhibit the visible bacterial growth after incubation at $37^{\circ} \mathrm{C}$ for 24 hours.

\subsection{Preparation of Chitosan-MWCNT}

Different amounts of purified MWCNTs were sonicated in chitosan solution (40 mg in $0.1 \mathrm{M}$ aqueous acetic acid, 
$40 \mathrm{~mL}$ ) for 20 mins and then stirred at room temperature for 8 hrs ${ }^{11}$.

The obtained chitosan-MWCNTs were collected and washed with ultra pure water by ultra centrifugation to remove unbound Chitosan, then collected and dried at room temperature to obtain Ch-MWCNTs with different CNT concentrations.

\section{Results}

\subsection{Morphological Analysis}

The morphology of MWCNT and its chitosan nanocomposites containing different percentage of MWCNT ranging from 1:50\% were investigated via TEM analysis using (JEM-1230-electron microscopy operated at 60 $\mathrm{KV}$ ) and presented in Figure 1. From the figure, it is clear that, there is a noticeable homogeneity between the modified MWCNT and chitosan and the modified MWCNT has a good distribution over the chitosan polymer surface where the homogeneity increased by increasing the percentage of CNT and. This result confirms the successful preparation of Chitosan-MWCNT nanocomposite.

\subsection{Fourier Transforms Infrared}

\section{Spectroscopy (FTIR)}

FTIR spectra of the chitosan and the nanocomposites of modified chitosan-MWCNTs recorded on a FTIR spectrophotometer (Thermo Nicolet, NEXUS, TM) in the range of $4000-400 \mathrm{~cm}^{-1}$ using $\mathrm{KBr}$ pellets.

Figure 2 illustrates FTIR for chitosan and ChitosanMWCNT nanocomposite, from the figure, it is obvious that, FTIR of chitosan was showed that the intense and wide band at $3220 \mathrm{~cm}^{-1}$ is attributed to the vibration of $-\mathrm{OH}$ group, the characteristic peaks at $1513 \mathrm{~cm}^{-1}$ is due to the vibration of $\mathrm{C}=\mathrm{O}$ while FTIR was showed the chitosan/CNT composite spectra, the intense bands at $3480 \mathrm{~cm}^{-1}$ and $1040 \mathrm{~cm}^{-1}$ are attributed to the stretching vibration of $\mathrm{N}-\mathrm{H}$ and $\mathrm{C}-\mathrm{O}$ respectively and also $\mathrm{C}=\mathrm{C}$ absorption at the wave number $2344 \mathrm{~cm}^{-1}$.

\subsection{Antimicrobial Activity}

The antibacterial activities of Ch, MWCNT and Ch-MWCNT nanocomposite were tested against four bacterial spp. by disc diffusion method and the results are shown in Table 1 and Figures 3 and 4. In general, all the nanocomposites Ch-MWCNT were more active than chitosan or MWCNT and exhibited higher antibacterial activity against the tested Gram positive and Gram negative bacteria. Inhibition zone ranged from 4 to $19 \mathrm{~mm}$ has been observed for tested bacteria. Nanocomposite (100:50 Ch-MWCNT) showed the best inhibitory activity against S. aureus (SA), E. coli (EC) and P. aeruginosa (PA) (inhibition zone 18.2, 14.5 and $19.2 \mathrm{~mm}$, respectively) and (100:25 Ch-MWCNT) against MRSA (inhibition zone $19.4 \mathrm{~mm}$ ). Nanocomposite (100:10 Ch-MWCNT) had the best activity against P. aeruginosa (inhibition zone $20.3 \mathrm{~mm}$ ).

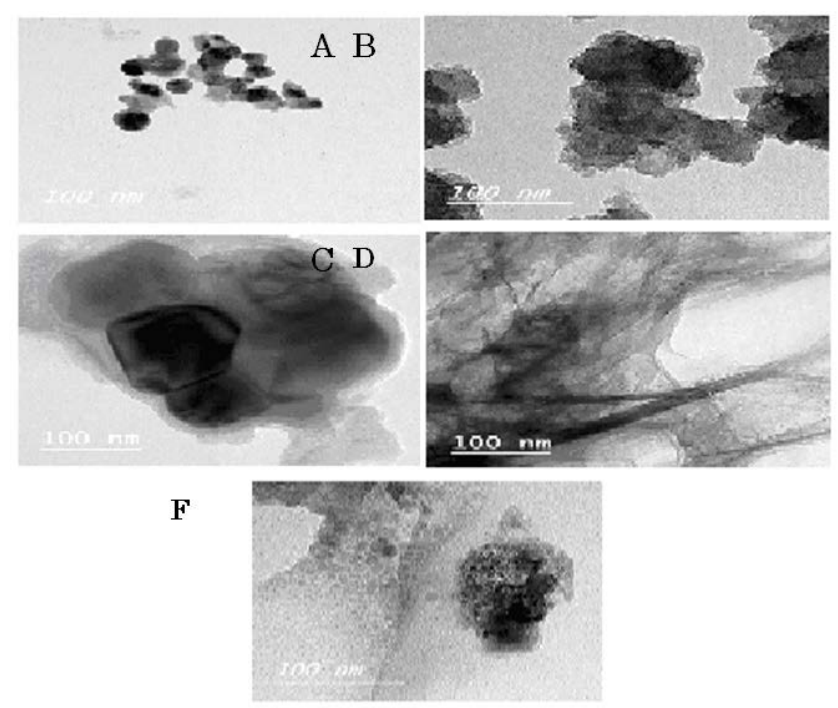

Figure 1. TEM imaged for Chitosan-MWCNT nanocomposite with different ratios of chitosan/CNT where A) $100 \mathrm{Ch}: \mathrm{CNT}, \mathrm{B}) 100 \mathrm{Ch}: 5 \mathrm{CNT}, \mathrm{C}) 100 \mathrm{Ch}: 10 \mathrm{CNT}, \mathrm{D})$ 100Ch:25CNT and E) 100Ch:50CNT.

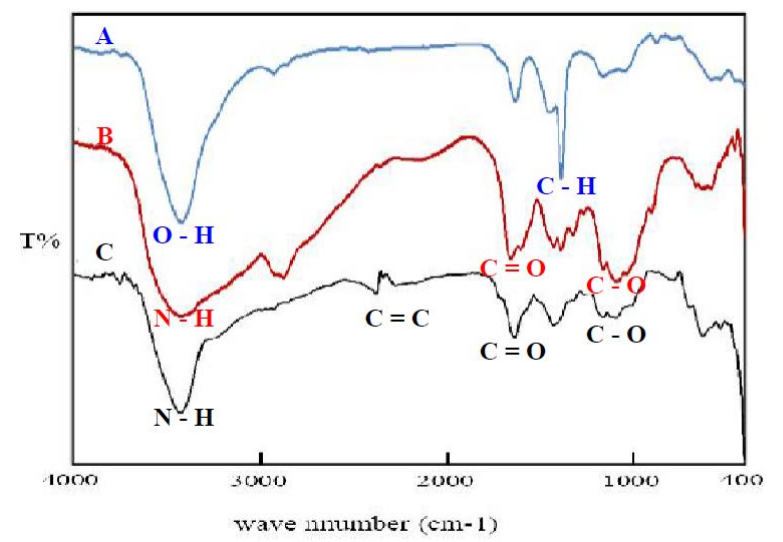

Figure 2. FTIR imaged for A) pure MWCNT, B) Pure Chitosan and C) modified Chitosan-MWCNT (100Ch:50CNT) nanocomposite. 
Compared with chitosan or MWCHT, all the Ch-MWCNT nanocomposites showed higher antimicrobial activity. The experiment examines that Ch-MWCNT nanocomposite showed greater antibacterial activity with respecting to increase the concentration towards MERS, S. aureus and E. coli when compared to P. aeruginosa. The concentration of Ch-MWCNT 100:50 nanocomposite show considerable activity toward the four tested microorganisms compare to other concentrations.

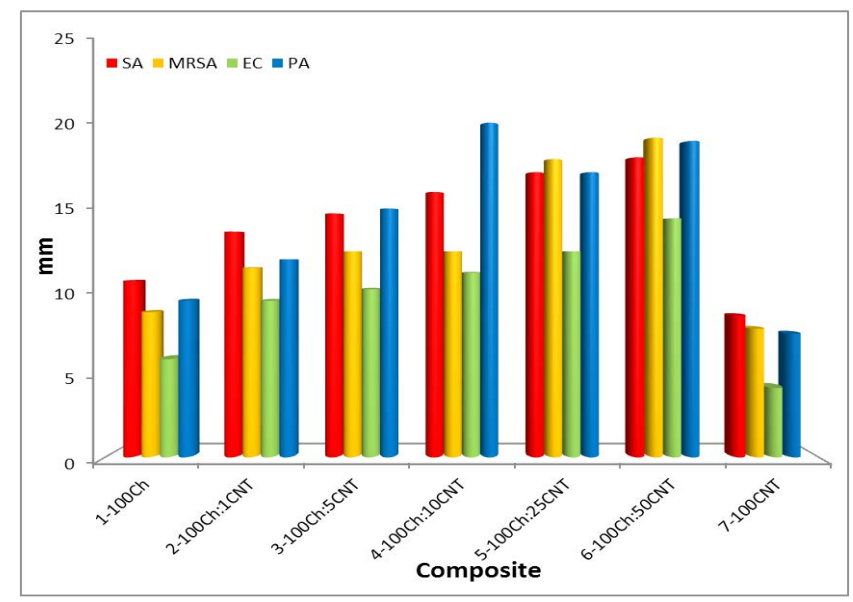

Figure 3. Antimicrobial activity of chitosan, MWCNT and different concentrations of Ch-MWCNT nanocomposites against tested pathogenic bacteria by disc diffusion method.

\subsection{Minimal Inhibitory Concentrations (MIC)}

Minimum Inhibitory Concentration (MIC) was regarded the minimal concentration of the Ch-MWCNT required to completely inhibit bacterial growth. The MIC values range from $0.0625 \mu \mathrm{l}$ to $0.5 \mu \mathrm{l}$. In this test Table 2, 4 test bacteria Staphylococcus aureus and Methicillin-resistant Staphylococcus aureus (MRSA) human pathogen Gram positive, Escherichia coli and P. aeruginosa human pathogen gram negative were used.
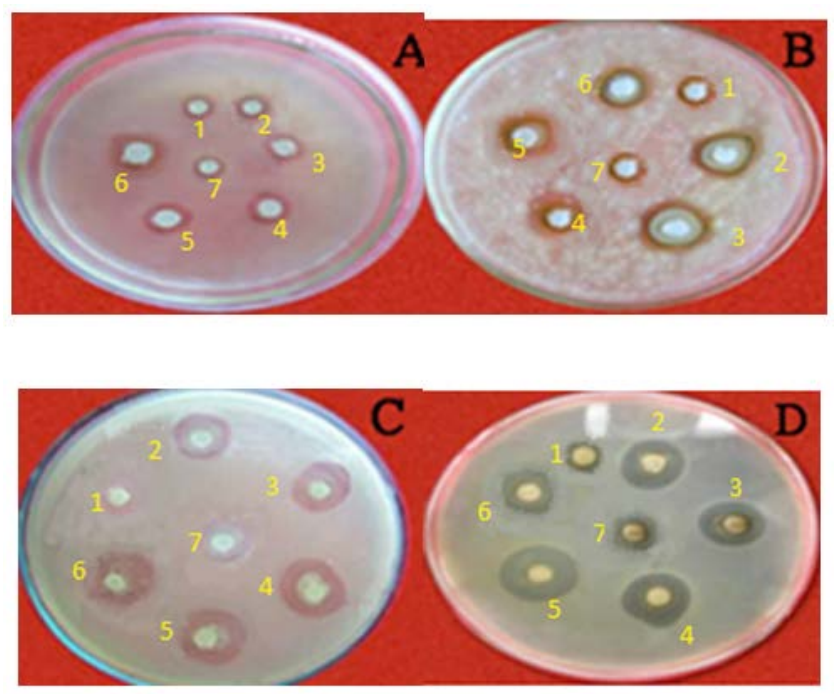

Figure 4. Inhibition zone $(\mathrm{mm})$ of chitosan, MWCNT and different concentrations of Ch-MWCNT nanocomposites against four tested pathogenic bacteria by disc diffusion method. A = MRSA, B = Staphylococcus aureus, $\mathrm{C}=\mathrm{E}$. coli, D $=$ P. aeruginosa. $(1=100 \mathrm{Ch}, 2=100 \mathrm{Ch}: 1 \mathrm{CNT}, 3=$ 100Ch:5CNT, $4=100 \mathrm{Ch}: 10 \mathrm{CNT}, 5=100 \mathrm{Ch}: 25 \mathrm{CNT}, 6=$ $100 \mathrm{CH}: 50 \mathrm{CNT}, 7=\mathrm{CNT}$ ).

Table 2 and Figure 5 showed that, the MIC of Ch-MWCNT (100:50 Ch-MWCNT) against Gram

Table 1. Antimicrobial activity of chitosan, MWCNT and different concentrations of Ch-

MWCNT nanocomposites against tested pathogenic bacteria by disc diffusion method

\begin{tabular}{|l|l|l|l|l|}
\hline \multirow{2}{*}{$\begin{array}{l}\text { Concentration } \\
\text { of Ch-MWCNT } \\
\text { nanocomposite } \\
(\mathrm{mg} / \mathrm{ml})\end{array}$} & \multicolumn{4}{|l}{ Diameter of inhibition Zone $(\mathrm{mm})$} \\
\cline { 2 - 5 } & S. aureus & MRSA & E. coli & P. aeruginosa \\
\hline $100 \mathrm{Ch}$ & $10.70 \pm 0.02$ & $8.80 \pm 0.02$ & $5.90 \pm 0.09$ & $9.51 \pm 0.04$ \\
\hline $100 \mathrm{Ch}: 1 \mathrm{CNT}$ & $13.70 \pm 0.08$ & $11.50 \pm 0.05$ & $9.50 \pm 0.04$ & $12.00 \pm 0.11$ \\
\hline $100 \mathrm{Ch}: 5 \mathrm{CNT}$ & $14.80 \pm 0.04$ & $12.50 \pm 0.05$ & $10.20 \pm 0.02$ & $15.10 \pm 0.5$ \\
\hline $100 \mathrm{Ch}: 10 \mathrm{CNT}$ & $16.10 \pm 0.06$ & $12.50 \pm 0.04$ & $11.20 \pm 0.11$ & $20.30 \pm 0.6$ \\
\hline $100 \mathrm{Ch}: 25 \mathrm{CNT}$ & $17.30 \pm 0.08$ & $18.10 \pm 0.01$ & $12.50 \pm 0.09$ & $17.30 \pm 0.4$ \\
\hline $100 \mathrm{Ch}: 50 \mathrm{CNT}$ & $18.20 \pm 0.05$ & $19.40 \pm 0.06$ & $14.50 \pm 0.08$ & $19.20 \pm 0.10$ \\
\hline $100 \mathrm{CNT}$ & $8.60 \pm 0.06$ & $7.80 \pm 0.08$ & $4.20 \pm 0.05$ & $7.50 \pm 0.7$ \\
\hline
\end{tabular}


Table 2. The minimum inhibitory concentration $(\mu \mathrm{g} / \mathrm{ml})$ of nanocomposite 100Ch:50CNT against the tested organisms

\begin{tabular}{|l|l|l|l|l|}
\hline \multirow{2}{*}{$\begin{array}{l}\text { Concentration } \\
\text { of 100Ch:50CNT } \\
\text { nanocomposite }(\mu \mathrm{g} / \mathrm{ml})\end{array}$} & \multicolumn{3}{|l|}{ MIC $(\mathrm{mm})$} \\
\cline { 2 - 5 } & Gram positive & \multicolumn{2}{l|}{ Gram negative } \\
\cline { 2 - 5 } & S. aureus & MRSA & E. coli & P. aeruginosa \\
\hline 0.5 & 0 & 0 & 0 & 0 \\
\hline 0.25 & 0 & 0 & + & 0 \\
\hline 0.125 & 0 & +++ & ++ & 0 \\
\hline 0.0625 & 0 & +++++ & +++ & 0 \\
\hline (+): Degree of growth &
\end{tabular}

negative and Gram positive pathogenic bacteria may be estimated between 0.5 and $0.0625 \mu \mathrm{g} / \mathrm{ml}$ and the effect of inhibition growth observe in conc. dependent species. In case of S. aureus, Ch-MWCNT shows the level of inhibition is high even in high concentration. As well as there is no antimicrobial activity in solution free from Ch-MWCNT which used as a vehicle control. This proves that antimicrobial activity is directly related to the Ch-MWCNT.
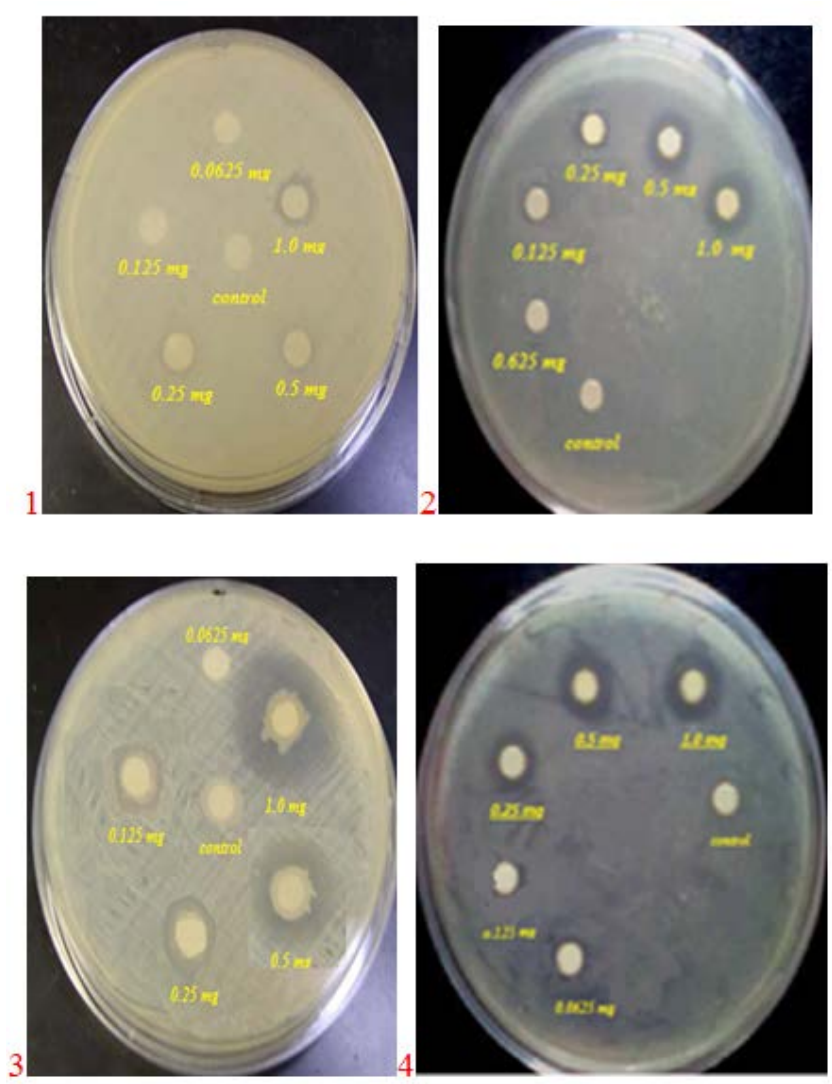

Figure 5. Some minimum inhibitory concentrations (mm) of the Ch-MWCNT (100:50) against the test organisms. (1= MRSA, 2 = Staphylococcus aureus, $3=\mathrm{E}$ coli, $4=\mathrm{P}$. aeruginosa).
Ch-MWCNT nanocomposite at 100:50 shows the highest activity against $\mathrm{S}$. aureus and $\mathrm{P}$. aeruginosa with an MIC less than $0.0625 \mu \mathrm{g} / \mathrm{ml}$. While the MIC for E. coli is $0.5 \mu \mathrm{g} / \mathrm{ml}$. The least MIC activity against MRSA is 0.25 Table 2. It shows that the extracts nanocomposites are more active against both Gram bacteria positive than Gram negative ones. Several mechanisms have been investigated to evaluate chitosan antimicrobial activity. The most accepted mechanism is that the chitosan positively charged combines with negative charge of bacterial cell membranes. The interaction takes place between protonated ammonium group in chitosan and the microbial cell membrane. This leads to promote the osmotic imbalance whereas CNT has a synergistic effect that damages the microbial membrane in situ in order to control the microbial growth.

\section{Discussion}

The antibacterial activities of $\mathrm{Ch}$, MWCNT and Ch-MWCNT nanocomposite are tested against four bacterial strains by disc diffusion method. Generally, all the nanocomposites Ch-MWCNT are more active than chitosan or MWCNT and exhibited a good antibacterial activity against the tested Gram negative and Gram positive bacteria. The MWCNT antimicrobial activity mechanism towards $\mathrm{S}$. aureus is simply by direct attachment of MWCNTs with the bacterial surface proteins.

The MWCNT emit from their nanocomposite targeting the bacteria. However, the presence of outer membrane has high net negative charge in E. coli becomes obstruct to MWCNT, which preventing it from binding to membranes initiating its antimicrobial activity. However, within $24 \mathrm{hrs}$ the microbial cell membrane lysis due to MWCNT attachment ${ }^{12,13}$. The inhibition mechanism 
of MRSA is depending on using chitosan with higher molecular weight which cause inhibition in the nutrient adsorption. On the other hand, chitosan with higher molecular weight might not much inhibit the Gram negative bacteria. This is due to the fact that higher molecular weight chitosan could not cause cell metabolism disorder. Our results are showed to be consequential with the previous studies ${ }^{14}$. Recently MWCNTs are confirmed to have strongly antimicrobial activity. Also MWCNTs relevant activities are referred to the nanodart behaviour with the suggested physical damage mechanism $\frac{15,16}{}$.

\section{Conclusion}

- Chitosan-Multi Walled Carbon Nanotube (Ch:MWCNT) nanocomposite with different chitosan: MWCNT ratios were successfully prepared.

- TEM analysis has shown that there is a noticeable homogeneity between the modified MWCNT and the chitosan polymer in the nanocomposite composed of 100 chitosan:50 CNT. In addition, the modified MWCNT has a good distribution of the chitosan polymer surfaces. This result confirms the successful preparation of ChitosanMWCNT nanocomposite.

- FTIR analysis imaging of chitosan, MWCNT and its chitosan nanocomposite shows that the presence of the carboxylic group, $\mathrm{C}=\mathrm{O}, \mathrm{N}-\mathrm{H}$ and $\mathrm{C}-\mathrm{O}$ respectively and also $\mathrm{C}=\mathrm{C}$ in the modified MWCNT.

- All the nanocomposites Ch-MWCNT were more active than chitosan or MWCNT and exhibited better antibacterial activities against the tested Gram positive and Gram negative bacteria. The concentration of Ch-MWCNT 100:50 nanocomposite show considerable activity toward the four tested microorganisms compares to other concentrations. Ch-MWCHT nanocomposite at 100:50 shows the highest activity against $\mathrm{S}$. aureus and Ps. aeruginosa with a MIC of 0.0625 $\mu \mathrm{g} / \mathrm{ml}$. While the MIC for MRSA and E. coli is $0.5 \mu \mathrm{g} / \mathrm{ml}$. The least MIC activity against MRSA and E. coil is 0.25 .

\section{References}

1. Iijima S. Synthesis of carbon nanotubes. Nature. 1991; 354:56-8. https://doi.org/10.1038/354056a0
2. Arkan E, Saber R, Karimi Z, Mostafaie A, Shamsipur M. Multi Wall Carbon Nanotube ionic liquid electrode modified with gold nanoparticles as a base for preparation of a novel impedimetric immunosensors for low level detction of human serum albumin in biological fluids. Journal of Pharmaceutical and Biomedical Analysis. 2014; 92:74-81. https://doi.org/10.1016/j.jpba.2014.01.005

3. Masoomi-Godarzi S, Khodadadi AA, Vesali-Naseh M, Mortazavi Y. Highly stable and selective non-enzymatic glucose biosensor using carbon nanotubes decorated by $\mathrm{Fe}_{3} \mathrm{O}_{4}$ nanoparticules. Journal of the Electrochemical Society. 2014; 161(1):B19-25. https://doi.org/10.1149/2.057401jes

4. Reza KK, Srivastava S, Yadav S, Biradar AM. Biofunctionalized carbon nanotubes platform for biomedical applications. Materials Letters. 2014; 126:126-30. https://doi.org/10.1016/j.matlet.2014.04.017

5. Twana MMW, Wing ML, Vitaliy V. Chitosan and its derivatives for application in mucoadhesive drug delivery systems. Polymers. 2018; 10:267-9. https://doi.org/10.3390/ polym 10030267

6. Gokarneshan N. Review article - Role of Chitosan in wound healing - a Review of the recent advances. Global Journal of Addiction and Rehabilitation Medicine. 2017; 4(3):1-10. https://doi.org/10.19080/GJARM.2017.04.555637

7. Difco Manual. 1969. https://www.trios.cz/wp-content/ uploads/sites/149/2016/08/DIFCO-A-BBL-MANUAL-2. pdf

8. Molecular cloning: A laboratory manual. 1989. https:// www.cshlpress.com/pdf/sample/2013/MC4/MC4FM.pdf

9. Biemer JJ. Antimicrobial susceptibility testing by the KirbyBauer disc diffusion method. Clinical Laboratory Science. 1973; 3(2):135-40.

10. Ruangpan L. Minimal Inhibitory Concentration (MIC) test and determination of antimicrobial resistant bacteria. Laboratory manual of standardized methods for antimicrobial sensitivity tests for bacteria isolated from aquatic animals and environment; 2004. p. 31-55.

11. Kang S, Mauter MS, Elimelech M. Physicochemical determinants of Multiwalled Carbon Nanotubebacterial cytotoxicity. Environmental Science and Technology. 2008; 42:7528-34. https://doi.org/10.1021/es8010173

12. Deokar AR, Lin LY, Chang CC, Ling YC. Single-walled carbon nanotube coated antibacterial paper: Preparation and mechanistic study. Journal of Materials Chemistry. 2013; 1:2639-46. https://doi.org/10.1039/c3tb20188k

13. Chen JH, Lu DQ, Chen B, Ouyang PK. Removal of U(VI) from aqueous solutions by using MWCNTs and chitosan modified MWCNTs. Journal of Radioanalytical and Nuclear Chemistry. 2013; 295:2233-41. https://doi.org/10.1007/ s10967-012-2276-y

14. Zheng LY, Zhu JF. Study on antimicrobial activity of chitosan with different molecular weights. Carbohydrate 
Polymers. 2003; 54:527-30. https://doi.org/10.1016/j. carbpol.2003.07.009a

15. Kang S, Pinault M, Pfefferle LD, Elimelech M. Single Walled Carbon Nanotubes exhibit strong antimicrobial activity. Langmuir. 2007; 23:8670-3. https://doi.org/10.1021/ la701067r
16. Lin N, Berton P, Moraes C, Rogers RD, Tufenkji N. Nanodarts, nanoblades and nanospikes: Mechanobactericidal nanostructures and where to find them. Advances in Colloid and Interface Science. 2017; 252:5568. https://doi.org/10.1016/j.cis.2017.12.007 